\title{
Costimulation with anti-cluster of differentiation 3 and anti-cluster of differentiation 28 reduces the activity of mucin 1-stimulated human mononuclear cells
}

\author{
STEPHEN E. WRIGHT ${ }^{1-4}$, KATHLEEN A. REWERS-FELKINS ${ }^{1}$, IMELDA QUINLIN ${ }^{1}$, \\ FATEMA ZOHRA $^{1}$ and JEWEL AHMED ${ }^{1}$ \\ ${ }^{1}$ Department of Internal Medicine, Women's Health Research Institute; \\ ${ }^{2}$ Department of Microbiology and Immunology, School of Medicine; ${ }^{3}$ Department of Biomedical Sciences, \\ School of Pharmacy, Texas Tech University Health Sciences Center; ${ }^{4}$ Harrington Cancer Center, Amarillo, TX 79106, USA
}

Received January 13, 2015; Accepted September 4, 2015

DOI: $10.3892 / \mathrm{ol} .2015 .3840$

\begin{abstract}
Cytotoxic T-lymphocyte activation and extension of the cell life span is necessary in order to enable immunotherapy to perform effectively against cancer. In the present study, mucin 1 (MUC1)-stimulated human mononuclear cells (M1SHMCs) were costimulated with bead-attached monoclonal antibodies specific for cluster of differentiation (CD)3 and CD28 receptors. The study was undertaken to determine whether costimulation was capable of enhancing the killing of cancer cells in vitro and of protecting non-obese diabetic severe combined immunodeficient mice from tumor development. Lysis of MCF-7 tumor cells by M1SHMCs was reduced following costimulation with anti-CD3 and anti-CD28. Furthermore, costimulation with anti-CD3 and anti-CD28 eliminated the protective effects of M1SHMCs on MCF-7 breast cancer cell growth in the non-obese diabetic severe combined immunodeficient mice. The present study suggested that costimulation with anti-CD3 and anti-CD28 is not advisable following antigen activation of lymphocytes under the conditions used here. Using a lower anti-CD3/CD28 bead to T-cell ratio may prevent immune suppression, however, further studies are required to support this hypothesis.
\end{abstract}

\section{Introduction}

Cytotoxic T-lymphocyte (CTL) activation and extension of the cell life span are necessary in order to enable immunotherapy to perform effectively against cancer cells (1). CTLs are

Correspondence to: Dr Stephen E. Wright, Department of Internal Medicine, Women's Health Research Institute, Texas Tech University Health Sciences Center, 1400 Wallace Boulevard, Amarillo, TX 79106, USA

E-mail: stephen.wright@ttuhsc.edu

Key words: anti-cluster of differentiation 3, anti-cluster of differentiation 28 , mucin 1 , mononuclear cells activated via the T-cell receptor (TCR; signal one) (2), which induces the proliferation of CTLs. Engagement of a second receptor, cluster of differentiation 28 (CD28; signal two), by ligands on antigen-presenting cells, is required to prevent anergy and the apoptosis of CTLs (3). This results in extension of the CTL lifespan. CTLs may be activated via the CD3 receptor [a component of the TCR (2)] and the CD28 receptor using monoclonal antibodies specific for their respective receptors (4). In order to obtain an adequate number of CTLs for the effective performance of immunotherapy, costimulation with anti-CD3 and anti-CD28 has been utilized (5). Costimulated lymphocytes cells have been demonstrated to exhibit logarithmic growth and inhibit apoptosis via enhanced cytotoxicity for the targeting of tumor cells (6). The present study aimed to determine whether the mucin 1 (MUC1)-stimulated human mononuclear cells (M1SHMCs) of breast cancer patients would demonstrate expanded levels of growth without compromising their ability to kill cancer cells when costimulated with anti-CD3 and anti-CD28.

\section{Materials and methods}

MUC1-variable number tandem repeat 1 (VNTR1) peptide. GSTAPPAHGVTSAPDTRPAP (7) peptide was synthesized by American Peptide Co., Inc., (Sunnyvale, CA, USA) and Novartis International AG (Basel, Switzerland).

Anti-CD3/CD28 antibody beads. Anti-CD28 antibodies were obtained from Murine Hybridoma 9.3 (8), which was a gift from Professor John Hansen (University of Washington, Seattle, USA). Dynabeads ${ }^{\circledR}$ M-450 Tosylactivated (Thermo Fisher Scientific, Inc., Waltham, MA, USA) are superparamagnetic polystyrene beads, which were activated using p-toluenesulfonyl chloride, according to the manufacturer's instructions. Anti-CD3 (OKT3; Beckman Coulter, Inc., Brea, CA, USA) and anti-CD28 (5 $\mu$ g each) were subsequently added to a centrifuge tube containing Buffer B Dynabeads kit solution. The tube was then placed in a Dynabeads ${ }^{\circledR}$ Rotator Mixer (Thermo Fisher Scientific, Inc.) and incubated for $24 \mathrm{~h}$ at $37^{\circ} \mathrm{C}$ with $5 \% \mathrm{CO}_{2}$. The following day, the tube was placed into the 
Dynal $^{\circledR}$ MPC-S magnet (Thermo Fisher Scientific, Inc.) for $3 \mathrm{~min}$. Supernatant was subsequently removed and discarded. The anti-CD3/anti-CD28-coated Dynabeads were washed twice in Buffer D [0.88 g NaCl, 0.1\% bovine serum albumin, $80 \mathrm{ml} 0.01 \mathrm{M}$ Na-phosphate $(\mathrm{pH} 7.4)]$ at $4^{\circ} \mathrm{C}$. Following each wash, the tube was placed into the Dynal magnet, and the supernatant was subsequently discarded. Buffer E [2X; $2.42 \mathrm{~g}$ Tris and $80 \mathrm{ml}$ distilled $\left.\mathrm{H}_{2} \mathrm{O}(\mathrm{pH} 8.5)\right]$ was added, and incubated overnight at $37^{\circ} \mathrm{C}$ with $5 \% \mathrm{CO}_{2}$. The following day Dynabeads were placed into the Dynal magnet, and the buffer was subsequently removed. The anti-CD28/anti-CD3-coated Dynabeads were then stored in AIM-V ${ }^{\circledR}$ medium (Gibco; Thermo Fisher Scientific, Inc.) at $-20^{\circ} \mathrm{C}$, for storage over one day, or at $4^{\circ} \mathrm{C}$ for less than one day.

Human cells. The present study was approved by the Institutional Review Board of Texas Tech University Health Sciences Center (Amarillo, TX, USA). All human cells were obtained from expired subjects in accordance with the Institutional Review Board criteria of the Texas Tech University Health Sciences Center. Frozen human peripheral blood hematopoietic stem cells were obtained from the Bone Marrow Transplant Laboratory of the Harrington Cancer Center (Amarillo, TX, USA) from expired, anonymous donors. Frozen human peripheral blood mononuclear cells (PBMCs) were obtained from the Bone Marrow Transplant Laboratory of the Harrington Cancer Center (Amarillo, TX, USA) by apheresis from an expired, anonymous donor with breast adenocarcinoma.

Cell culture conditions. Procedures were performed as previously described (9). Mononuclear cells were not human leukocyte antigen typed, as our previous studies (10-12) and another literature study (13) identified that cytotoxicity by M1SHMC may be non-major histocompatibility complex-restricted. The cells were cultured at $2 \times 10^{6}$ cells $/ \mathrm{ml}$ in AIM V ${ }^{\circledR}$ serum-free lymphocyte medium (Gibco; Thermo Fisher Scientific, Inc.) and maintained in a $37^{\circ} \mathrm{C}$ humidified $5 \% \mathrm{CO}_{2}$ atmosphere (14). Interleukin-2 (100 IU/ml; Novartis International AG) was added twice/week. The cells were stimulated with MUC1-VNTR1 peptide on days zero and seven, at $1 \mu \mathrm{g} / \mathrm{ml}$. The cells were harvested on day eight.

Costimulation of mononuclear cells. M1SHMCs $\left(2.0 \times 10^{7}\right.$ cells $)$ were costimulated by anti-CD3/anti-CD28 beads according to the manufacturer's instructions, at a 3:1 ratio of beads to cells (6) in AIM V ${ }^{\circledR}$ medium. This occurred at the intervals shown in Fig. 1 (once, twice or three times/week) or three times/week, as shown in Figs. 2-4 (days 9, 11 and 13, with harvesting on day 14).

Growth of MCF-7 cell line. Human breast carcinoma MCF-7 cells were utilized as the target cells (15). The cells were cultured in Dulbecco's modified Eagle's medium (DMEM; Gibco; Thermo Fisher Scientific, Inc.) supplemented with $10 \%$ fetal bovine serum (Hyclone, Logan, UT, USA), $1 \%$ bovine insulin (Gibco Life Technologies) and 1\% L-glutamine (Gibco; Thermo Fisher Scientific, Inc.) at $37^{\circ} \mathrm{C}$ with $5 \% \mathrm{CO}_{2}$. Medium was replaced twice/week by removal of the DMEM and addition of $15 \mathrm{ml}$ fresh DMEM to the flask.

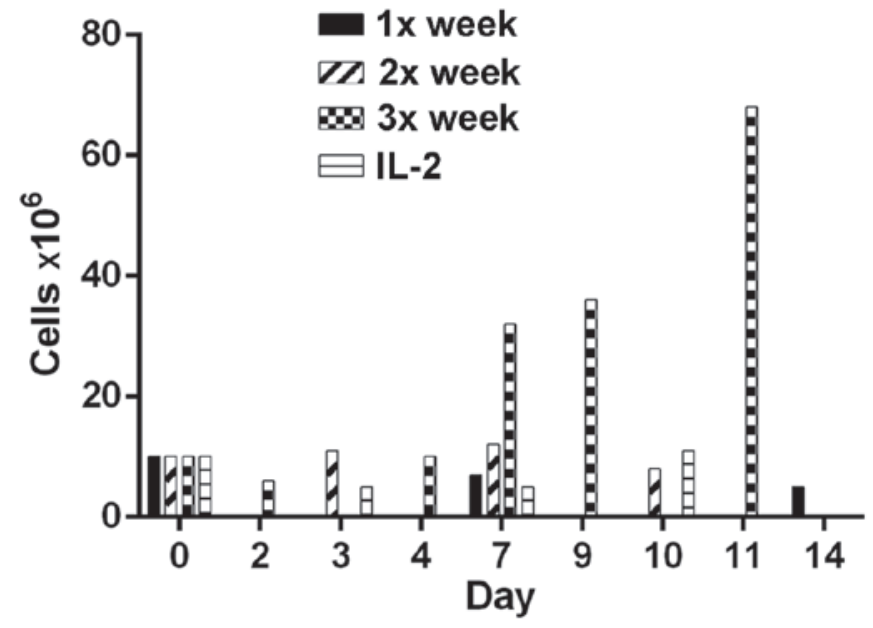

Figure 1. Growth of peripheral blood mononuclear cells with anti-CD3/anti-CD28 costimulation. A total of $2.0 \times 10^{7}$ mononuclear cells were costimulated with anti-CD3/anti-CD28 beads at a 3:1 ratio of beads to cells at the intervals of once, twice or three times/week. CD, cluster of differentiation; IL, interleukin. Each bar represents a single culture, thus no statistical analysis was performed.

Chromium release assays. The MCF-7 cells were used as targets in a Chromium 51 release assay (16). The cells were labeled with $\left[{ }^{51} \mathrm{Cr}\right]$ sodium chromate $\left(200 \mu \mathrm{Ci}\right.$ per $1 \times 10^{7}$ cells; New England Nuclear, PerkinElmer, Inc., Waltham, MA, USA) and added to microtiter plates at a concentration of $5 \times 10^{3}$ target cells/well. Effector cells were tested at the effector to target cell ratios of 2.5, 5.0 and 10, indicated in figures 2 and 3 . DMEM was added instead of effector cells to the spontaneous ${ }^{51} \mathrm{Cr}$ release control wells. The maximum target control wells had 2\% Triton X-100 (Sigma-Aldrich, St. Louis, MO, USA) added in place of the effector cells, in order to lyse the target cells. Assays were incubated for $18 \mathrm{~h}$, which has been identified to be superior to $4 \mathrm{~h}(9)$, at $37^{\circ} \mathrm{C}$ with $5 \% \mathrm{CO}_{2}$, and each assay was performed in triplicate. Plates were centrifuged at $200 \mathrm{x} \mathrm{g}$, and one half of the $100 \mu \mathrm{l}$ supernatant was harvested. Radioactivity released into the supernatant was measured using liquid scintillation or $\gamma$ counting. The specific percentage lysis was calculated using the formula below, where cpm represents counts/minute:

$$
\% \text { lysis }=\frac{(\text { mean experimental cpm }- \text { mean spontaneous } \mathrm{cpm})}{(\text { mean maximum } \mathrm{cpm}-\text { mean spontaneous } \mathrm{cpm})} \times 100
$$

In vivo protection experiment. Animal care was in accordance with the guidelines of Texas Tech University Health Sciences Center (Amarillo, TX USA). Mice were housed in individually ventilated cages supplied HEPA filtered air, with a maximum of five mice per cage. Each animal holding room was supplied with 10-15 air changes per hour, and pressurized relative to the immunocompetance of the animals. The light/dark cycle was set from 07:00-19:00 (light cycle- low intensity). Diet and water was ad libitum, and the water was de-ionized and autoclaved. The immunodeficient diet was Global Irradiated Code 2918 (Harlan Teklad Labs, Houston, TX USA). Mice were housed at a temperature of $68-79^{\circ} \mathrm{F}$ and humidity between $30-70 \%$. Female non-obese diabetic severe combined immunodeficient mice (Jackson Laboratory, Bar Harbor, ME, USA), at 6-12 weeks of age, were injected subcutaneously (SC) 


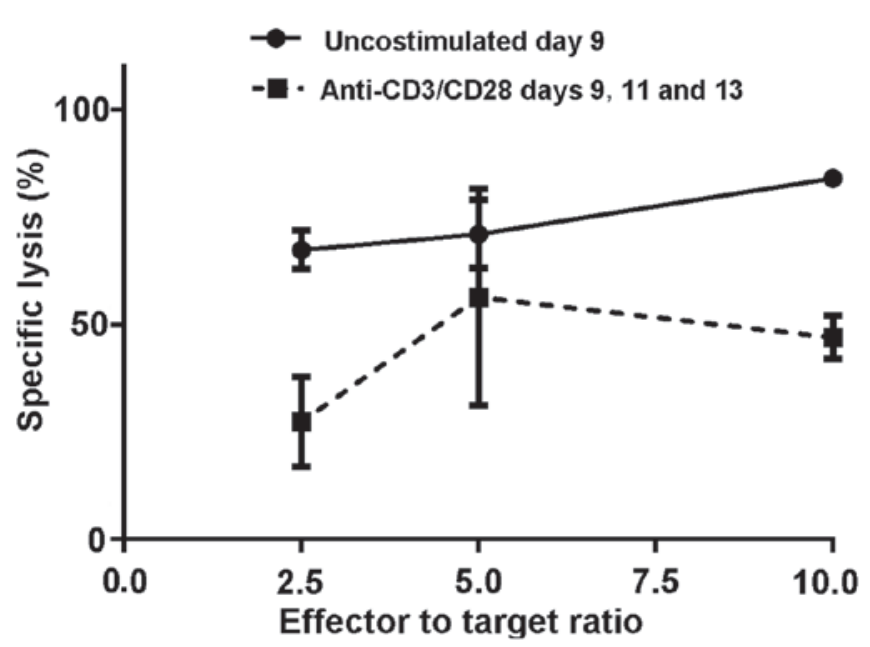

Figure 2. Specific lysis of MCF-7 cells by M1SHMCs vs. M1SHMCs costimulated with anti-CD3/anti-CD28 beads at the indicated effector to target ratio Peripheral blood mononuclear cells were stimulated twice by mucin 1 (days 1 and 8). M1SHMCs were then costimulated with anti-CD3/anti-CD28 beads on days 9, 11 and 13, and harvested on day 14. Data are expressed as the mean \pm standard error $(\mathrm{P}=0.004$, two-tailed $t$ test for $\mathrm{E}$ : $\mathrm{T}$ of 2.5 : 1$)$. Certain bars are masked by the symbols. M1SHMC, mucin 1-stimulated human mononuclear cells; $\mathrm{CD}$, cluster of differentiation.

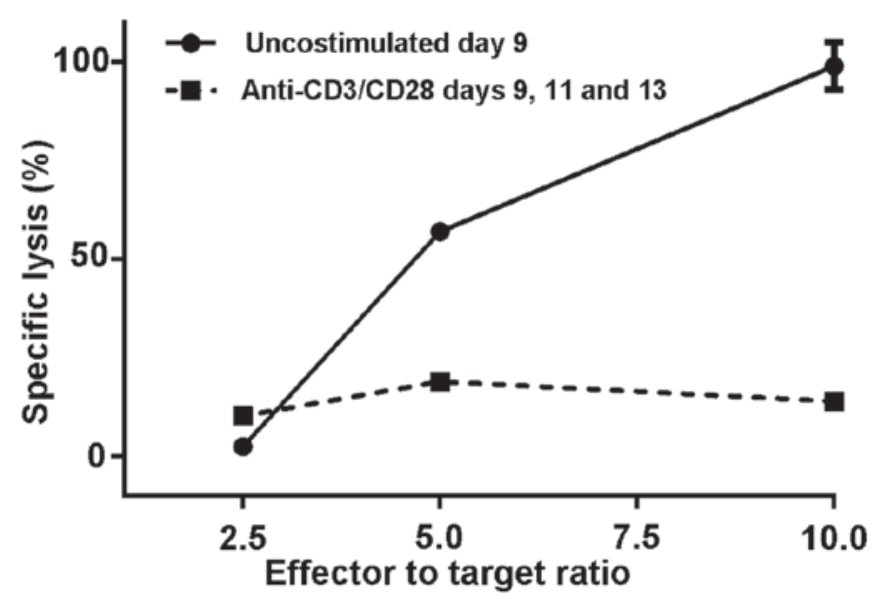

Figure 3. Specific lysis of MCF-7 cells by M1SHMCs vs. M1SHMCs costimulated with anti-CD3/anti-CD28 beads at the indicated effector to target ratio. Peripheral blood mononuclear cells were stimulated twice by mucin 1 (days 1 and 8). M1SHMCs were then costimulated with anti-CD3/anti-CD28 beads on days 9, 11 and 13, and harvested on day 14. Data are expressed as the mean \pm standard error, $99 \% \pm 3$ vs. $14 \% \pm 0(\mathrm{P}=0.0001$, two-tailed t test $)$ for E:T of 10:1). Certain bars are masked by the symbols. M1SHMC, mucin 1-stimulated human mononuclear cells; $\mathrm{CD}$, cluster of differentiation.

in the back of the neck, with $0.1 \mathrm{ml}$ phosphate-buffered saline (PBS):Matrigel at a 1:1 ratio (Gibco; Thermo Fisher

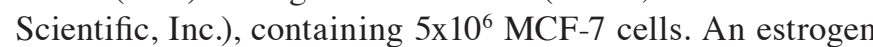
pellet $(0.18 \mathrm{mg}, 60$ day release; Innovative Research of America, Sarasota, FL, USA) was injected SC into the posterior back of the mouse to allow tumor growth. PBS, or anti-CD3/CD28 antibody beads or $5 \times 10^{7}$ M1SHMC were injected intraperitoneally (IP) on the same day as the tumor cells. Control animals received PBS or anti-CD3/CD28 antibody beads individually. The first group of 7 mice received MCF-7; the second group of 1 mouse received anti-CD3/CD28 antibody beads IP; the third group of 4 mice received $5 \times 10^{7}$ M1SHMC

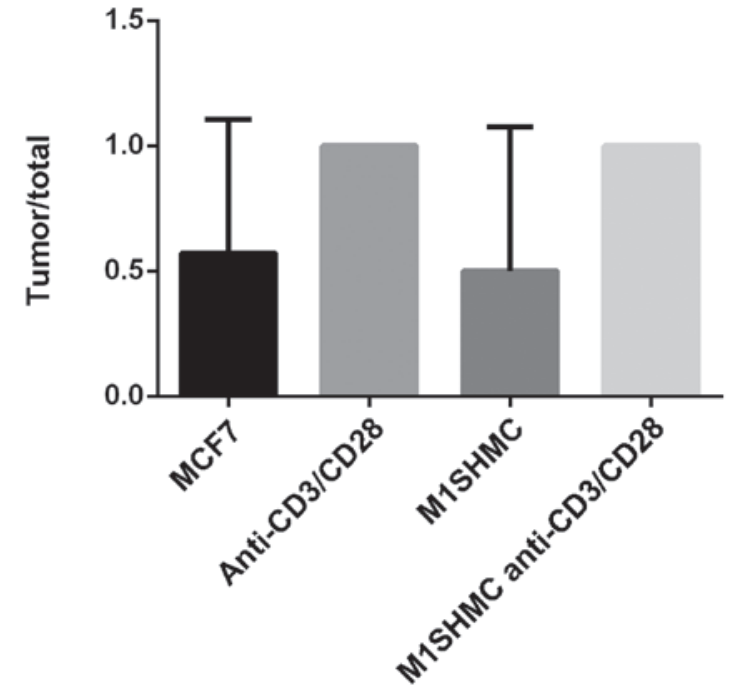

Figure 4. Effect of costimulation with anti-CD3/anti-CD28 on the inhibition of tumor production by M1SHMCs. M1SHMCs are peripheral blood mononuclear cells that were stimulated twice by mucin 1 (on days 1 and 8). M1SHMC were then costimulated with anti-CD3/anti-CD28 beads on days 9, 11 and 13, and harvested on day 14. Female non-obese diabetic severe combined immunodeficient mice were injected SC with $5 \times 10^{6} \mathrm{MCF}-7$ tumor cells in the right posterior neck (on day 0). An estrogen pellet was injected SC at the same time, but at an alternative site to the tumor cells, to allow tumor growth. On the same day that tumor cells were injected IP, control animals were injected with phosphate-buffered saline or the anti-CD3/CD28 antibody beads individually. The first group of 7 mice received MCF-7; the second group of 1 mouse received anti-CD3/CD28 antibody beads IP; the third group of 4 mice received $5 \times 10^{7}$ M1SHMC IP; and the fourth group of 4 mice received M1SHMC and anti-CD3/CD28 antibody beads. Each mouse was examined for tumor development three times/week for one month. The observed tumor development at 28 days post-injection was used for statistical analysis. Data are expressed as the mean \pm standard error $\mathrm{P}=0.4$ (Chi-square) group 3 vs. group 4 . Certain bars are masked by the symbols. The Y axis is the fraction of the number of mice with tumors divided by the total number of mice injected. CD, cluster of differentiation; M1SHMC, mucin 1 stimulated human mononuclear cells; SC, subcutaneously; IP, intraperitoneally.

IP; and the fourth group of 4 mice received M1SHMC and anti-CD3/CD28 antibody beads. Each mouse was examined for tumor development three times/week for one month. Observed tumor development at 28 days post-injection was used for statistical analysis. . The present study adhered to the Guidelines for Ethical Conduct in the Care and Use of Animals (https://www.aaalac.org/resources/theguide.cfm) detailed by the American Psychological Association Board of Scientific Affairs Committee on Animal Research and Ethics.

Statistical analysis. GraphPad Prism 6 (GraphPad Software Inc., La Jolla, CA, USA) statistical software package was utilized to analyze the data. Data are expressed as the mean \pm standard error. ANOVA, Chi-square, and two-tailed Student's t test statistical analyses were performed. $\mathrm{P}<0.05$ was used to indicate statistical significance.

\section{Results}

Growth of peripheral blood mononuclear cells with anti-CD3/anti-CD28 costimulation. Monoclonal antibodies were bound onto beads in order to costimulate patient lymphocytes. Cells underwent costimulation by anti-CD28/anti-CD3 
Dynabeads one, two or three times/week. Cells costimulated three times/week demonstrated optimal proliferation and an increased cell count, from 10 to 68 million (Fig. 1). Costimulation once or twice/week did not significantly increase the cell number. Each bar represents a single culture, thus no statistical analysis was performed. Thus, M1SHMC were costimulated three times with anti-CD3/anti-CD28 beads on days 9,11 and 13, and harvested on day 14 .

Specific lysis of MCF-7 cells by MISHMCs was compared to M1SHMCs costimulated with anti-CD3/anti-CD28 beads at the indicated effector to target ratio. M1SHMCs were then compared with costimulated M1SHMCs using an in vitro MCF-7 cell-killing assay and in an in vivo MCF-7 tumor formation inhibition experiment. Costimulation of M1SHMC with anti-CD3/anti-CD28 beads did not result in enhanced killing of the MCF-7 cells. Reduced killing of MCF-7 cells was observed in two separate experiments (Figs. 2 and 3). In the first experiment, at a 10:1 ratio of effector to target cells, the M1SHMCs possessed a specific lysis rate of $67 \pm 3 \%$ vs. $27 \pm 6 \%$ ( $\mathrm{P}=0.004$, two-tailed $\mathrm{t}$ test) for the anti-CD3/anti-CD28 bead costimulated M1SHMCs (Fig. 2). In the second experiment, the observed difference was even greater. At a 10:1 ratio of effector to target cells, the M1SHMCs possessed a specific lysis rate of $99 \pm 3 \%$ vs. $14 \pm 0 \%+0(\mathrm{P}<0.0001$, two-tailed t test $)$ for the anti-CD3/anti-CD28 bead costimulated M1SHMC (Fig. 3).

Effect of costimulation with anti-CD3/anti-CD28 on the inhibition of tumor production by MISHMCs. The in vivo results were consistent with those observed in vitro. There was enhanced tumor formation in the animals injected with breast cancer patients PBMCs, costimulated with anti-CD3 and anti-CD28 beads with or without MUC1 stimulation (Fig. 4). In the first group of 7 mice that received MCF-7, 4 developed tumors (57\%); in the second group of 1 mouse that received anti-CD3/CD28 antibody beads, a tumor developed (100\%); in the third group of 4 mice that received M1SHMC, two mice developed tumors (50\%); and in the fourth group of 4 mice that received M1SHMC and anti-CD3/CD28 antibody beads, all mice developed tumors (100\%; $\mathrm{P}=0.4$ using Chi-square, group 3 vs. group 4).

\section{Discussion}

This present study was performed in order to investigate the optimum interval of time for the costimulation of M1SHMCs with anti-CD3/CD28 antibody beads, to promote the proliferation of CTLs and the killing of breast cancer cells, thereby preventing tumor growth. With regard to lymphocyte cell growth, the most frequent intervals of costimulation with anti-CD3/CD28 antibody beads provided the optimal rate of cell proliferation. However, the anti-CD3/CD28 bead costimulation of M1SHMCs resulted in a significant decrease in breast cancer cell killing activity. This led to enhanced tumor cell growth. Whilst costimulation with anti-CD3/CD28 antibody beads may be utilized for the activation of lymphocytes (17), the results of the present study suggested that costimulated M1SHMCs, whilst exhibiting higher rates of proliferation, possess a reduced ability to kill cancer cells, and thus this method of treatment may not be advisable following antigen activation of lymphocytes under the conditions used here. We have previously shown that continued stimulation of CTL rendered them anergic (9). In support of this, constitutive proliferating CAR T cells showed inferior antitumor effect (18). In addition, repetitive signaling rendered CAR T cells susceptible to activation -induced cell death (AICD) (19).

In conclusion, whilst CTL activation and extension of the cell life span may be necessary in order to enable immunotherapy to perform effectively against cancer cells (1), excessive proliferation and signaling of the $\mathrm{T}$ cells may inhibit their antitumor activity. This resulting immune suppression may be prevented by using a lower anti-CD3/CD28 bead: T-cell ratio (20), which should reduce the $\mathrm{T}$ cells signaling through the CD3 complex, and reduce activation -induced cell death. Another alternative is altering the anti-CD3/CD28 ratios (21), where a lower anti-CD3/CD28 ratio should reduce activation -induced cell death and reduce apoptosis through CD28 engagement. A more physiological method that could be utilized for costimulation may be artificial antigen-presenting cells $(22,23)$, with the addition of additional costimulatory and pro-survival molecules.

\section{Acknowledgements}

The authors would like to thank the Coffee Memorial Blood Center (Amarillo, TX, USA) and the Harrington Cancer Center for providing the PBMCs, and those mentioned in the text for providing materials and/or services. Deena C. Victor, research assistant, Texas Tech University Health Sciences Center, participated in the initial phase of the studies. The present study was supported in part by VA Medical Research funds (0006), Harrington Research Foundation (Amarillo, TX, USA) and the Women's Health Research Institute (Texas Tech University Health Sciences Center).

\section{References}

1. Barrett DM, Singh N, Porter DL, Grupp SA and June CH: Chimeric antigen receptor therapy for cancer. Annu Rev Med 65: 333-347, 2014.

2. Lechler R and George AJT: 21. Transplantation and rejection: Immunology. In: Roitt I, Bristoff $\mathbf{J}$ and Male D (eds): Immunology. 5th edition. Mosby, Philadelphia, NJ, 1998.

3. Boise LH, Minn AJ, Noel PJ, et al: CD28 costimulation can promote T cell survival by enhancing the expression of Bcl-XL. Immunity 3: 87-98, 1995.

4. Riddell SR and Greenberg PD: The use of anti-CD3 and anti-CD28 monoclonal antibodies to clone and expand human antigen-specific T cells. J Immunol Methods 128: 189-201, 1990.

5. Levine BL, Ueda Y, Craighead N, Huang ML and June CH: CD28 ligands CD80 (B7-1) and CD86 (B7-2) induce long-term autocrine growth of $\mathrm{CD}^{+} \mathrm{T}$ cells and induce similar patterns of cytokine secretion in vitro. Int Immunol 7: 891-904, 1995.

6. Levine BL, Cotte J, Small CC, et al: Large-scale production of CD4+ T cells from HIV-1-infected donors after CD3/CD28 costimulation. J Hematother 7: 437-448, 1998.

7. Gendler S, Taylor-Papadimitriou J, Duhig T, Rothbard J and Burchell J: A highly immunogenic region of a human polymorphic epithelial mucin expressed by carcinomas is made up of tandem repeats. J Biol Chem 263: 12820-12823, 1988

8. Hansen J, Martin P and Nowinski R: Monoclonal antibodies identifying a novel T-Cell antigen and Ia antigens of human lymphocytes. Immunogenetics 10: 247-260, 1980.

9. Wright SE, KhaznadarR, Wang Z, Quinlin IS, Rewers-Felkins KA, Phillips CA and Patel S: Generation of MUC1-stimulated mononuclear cells using optimized conditions. Scand J Immunol 67: 24-29, 2008. 
10. Wright SE, Rewers-Felkins KA, Quinlin IS, Eldridge PW, Zorsky PE, Klug PP, Phillips CA and Philip R: Adoptive immunotherapy of mucin 1 expressing adenocarcinomas with mucin1 stimulated human peripheral blood mononuclear cells. Int J Mol Med 9: 401-404, 2002.

11. Wright SE, Kilinski L, Talib S, Lowe KE, Burnside JS, Wu JY, Dolby N, Dombrowski KE, Lebkowski JS and Philip R: Cytotoxic T lymphocytes from humans with adenocarcinomas stimulated by native MUC1 mucin and a mucin peptide mutated at a glycosylation site. J Immunother 23: 2-10, 2000.

12. Wright SE, Rewers-FelkinsKA, Quinlin IS,Fogler WE,Phillips CA Townsend M, Robinson W and Philip R: MHC-unrestricted lysis of MUC1-expressing cells by human peripheral blood mononuclear cells. Immunol Invest 37: 215-225, 2008

13. Alajez NM, Schmielau J, Alter MD, Cascio M and Finn OJ: Therapeutic potential of a tumor-specific, MHC-unrestricted T-cell receptor expressed on effector cells of the innate and the adaptive immune system through bone marrow transduction and immune reconstitution. Blood 105: 4583-4589, 2005.

14. Kanof ME and Smith PD: Preparation of human mononuclear cell populations and subpopulations. In: Current Protocols in Immunology. Coligan JE (ed) John Wiley \& Sons, Inc. New York, NY, USA, p7, 1994

15. Zhai YF, Esselman WJ, Oakley CS, Chang CC and Welsch CW Growth of MCF-7 human breast carcinoma in severe combined immunodeficient mice: Growth suppression by recombinant interleukin-2 treatment and role of lymphokine-activated killer cells. Cancer Immunol Immunother 35: 237-245, 1992.

16. Wunderlich J: Chromium Release Cytoxicity Assay. In: Current Protocols in Immunology. Coligan JE (ed) John Wiley \& Sons, Inc., New York, NY, USA, p3, 1994.

17. Levine BL, Bernstein WB, Connors M, Craighead N, Lindsten T, Thompson CB and June CH: Effects of CD28 costimulation on long-term proliferation of CD4+ T cells in the absence of exogenous feeder cells. J Immunol 159: 5921-5930, 1997.
18. Frigault MJ, Lee J, Basil MC, Carpenito C, Motohashi S, Scholler J, Kawalekar OU, Guedan S, McGettigan SE, Posey AD $\mathrm{Jr}$, et al: Identification of chimeric antigen receptors that mediate constitutive or inducible proliferation of T cells. Cancer Immunol Res 3: 356-367, 2015.

19. Künkele A, Johnson AJ, Rolczynski LS, Chang CA, Hoglund V, Kelly-Spratt KS and Jensen MC: Functional Tuning of CARs Reveals Signaling Threshold above Which CD8+ CTL Antitumor Potency Is Attenuated due to Cell Fas-FasL-Dependent AICD. Cancer Immunol Res 3: 368-379, 2015.

20. Kalamasz D, Long SA, Taniguchi R, Buckner JH, Berenson RJ and Bonyhadi M: Optimization of human T-cell expansion ex vivo using magnetic beads conjugated with anti-CD3 and Anti-CD28 antibodies. J Immunother 27: 405-418, 2004.

21. Li Y and Kurlander RJ: Comparison of anti-CD3 and anti-CD28-coated beads with soluble anti-CD3 for expanding human T cells: Differing impact on CD8 T cell phenotype and responsiveness to restimulation. J Transl Med 8: 104, 2010.

22. Maus MV, Thomas AK, Leonard DGB, Allman D, Addya K, Schlienger K, Riley JL and June CH: Ex vivo expansion of polyclonal and antigen-specific cytotoxic T lymphocytes by artificial APCs expressing ligands for the T-cell receptor, CD28 and 4-1BB. Nat Biotechnol 20: 143-148, 2002.

23. Singh H, Figliola MJ, Dawson MJ, Olivares S, Zhang L, Yang G, Maiti S, Manuri P, Senyukov V, Jena B, et al: Manufacture of clinical-grade CD19-specific T cells stably expressing chimeric antigen receptor using Sleeping Beauty system and artificial antigen presenting cells. PLoS One 8: e64138, 2013. 